\title{
Implications of exact SUSY gauge couplings for QCD
}

\author{
L. Randall \\ MIT, Cambridge Massachusetts 02139 \\ and Theory Division, CERN CH-1211, Geneva 23, Switzerland \\ R. Rattazzi \\ Theory Division, CERN CH-1211, Geneva 23, Switzerland \\ E. Shuryak \\ MIT, Cambridge, Massachusetts 02139
}

and Department of Physics and Astronomy, State University of New York at Stony Brook, New York 11794-3800

(Received 19 May 1998; published 11 January 1999)

\begin{abstract}
The phase structure of SUSY gauge theories can be very different from their nonsupersymmetric counterparts. Nonetheless, there is interesting information which might be gleaned from a detailed investigation of these theories. In particular, we study the precise meaning of the strong interaction scale $\Lambda$. We ask whether one can meaningfully apply naive dimensional analysis and also ask whether the study of supersymmetric theories can shed light on the apparent discrepancy between the perturbative scale $\Lambda_{Q C D}$ and the "chiral Lagrangian", scale $\Lambda_{\chi}$. We show that in $N=1$ supersymmetric Yang-Mills theory, "naive dimensional analysis" seems to work well, with $\Lambda_{\chi}$ consistently equal to the scale at which the perturbatively evolved physical coupling becomes of order $4 \pi$. We turn to $N=2$ theories to understand better the effect of instantons in accounting for the QCD discrepancy between scales. In $N=2$ supersymmetric $S U(2)$ the instanton corrections are known to all orders from the Seiberg-Witten solution and give rise to a finite scale ratio between the scale at which the perturbatively evolved and "nonperturbatively evolved" couplings blow up. Correspondingly, instanton effects are important even when the associated perturbatively evolved gauge coupling only gives $\alpha$ of order 1 (rather than $4 \pi$ ). We compare the $N=2$ result to instanton-induced corrections in QCD, evaluated using lattice data and the instanton liquid model, and find a remarkably similar behavior.

[S0556-2821(99)02201-8]
\end{abstract}

PACS number(s): 12.60.Jv, 11.30.Pb

\section{INTRODUCTION}

One usually employs the notion of a "chiral Lagrangian" or sigma model to describe the low-energy degrees of freedom of a strongly interacting gauge theory below a scale which we will refer to as $\Lambda_{N D A}$, where $\Lambda_{N D A}$ sets the scale for the cutoff and the suppression of higher derivative interactions, with coefficients determined by "naive dimensional analysis" (NDA) [1]. Although there is a potential ambiguity in its precise definition, one would expect $\Lambda_{N D A}$ to be the point where the original perturbative formulation of the field theory becomes impossible, so that perturbation theory requires the use of effective degrees of freedom and effective Lagrangians. The well-studied example of this phenomenon is QCD, where the cutoff scale for the chiral Lagrangian is of order $1 \mathrm{GeV}$.

It can be argued that the $\Lambda_{N D A}$ scale should be the scale at which perturbation theory fails completely in the sense that a coupling is of order $4 \pi$ so that a loop expansion is no longer possible [2]. However, in QCD this higher scale is mysterious, as the running QCD coupling should be of order $\alpha$ $\sim 4 \pi$ at the $\Lambda_{N D A}$ scale of order $1 \mathrm{GeV}$. According to the perturbative evolution, this is manifestly not the case. Related to this puzzle is the question of why the $1 \mathrm{GeV}$ scale is relevant at all, in light of the fact that both scales at which the coupling blows up $\left(\Lambda_{\text {pert }}\right)$ and the confinement scales $\left(\Lambda_{\text {conf }}\right)$ are of order $250 \mathrm{MeV}$. Although one might take the position that order of magnitude estimates might not lead to a better understanding, the whole point of naive dimensional analysis is to include as well as possible any large factors (of order $4 \pi$ ) which can be readily identified. We will discuss some exact results in supersymmetric (SUSY) theories to see whether they give some insight into this discrepancy between scales. This might seem absurd in that the phase structure of supersymmetric theories can differ qualitatively from a nonsupersymmetric theory. However, there are some questions which one has about QCD that can be meaningfully asked about solvable supersymmetric theories. These include the question of whether or not there can exist two distinct meaningful physical scales, whether or not the coupling becomes nonperturbative at one or the other of these scales, and which nonperturbative effects are significant at any given scale.

We will show that in $N=1$ super Yang-Mills (SYM) theory the scale at which the perturbative expansion breaks down according to the all order beta function is $\sim\left(8 \pi^{2} N\right)^{1 / 3}$ bigger than the scale at which the holomorphic coupling blows up. We will show that the larger scale is in qualitative agreement with the NDA scale as determined from the exact gluino condensate. The basic conclusion is that there is one scale which determines the physics. This scale is the NDA scale. However, the fact that the perturbatively evaluated coupling blows up at the NDA scale is not the same as the behavior of QCD, where the NDA scale is not associated 
with the perturbative blowup of the coupling. It does however suggest that NDA is a reliable tool that can be reasonably applied to estimate Kahler potential terms in phenomenological applications of strongly coupled supersymmetric theories.

We then argue that the mismatch of scales in QCD could be due to nonperturbative corrections. That these effects can be large even when the perturbative coupling is relatively small will be shown using the exact results from $N=2 \mathrm{SU}(2)$ in Sec. III. In Sec. IV we will veer from exact results back to QCD. We use the instanton-induced corrections to the effective charge defined by Callan, Dashen and Gross (CDG) [3], which includes integrating out instantons of sizes less than some $\rho_{\max }$. Those integrals are done using available lattice data for $\mathrm{SU}(2), \mathrm{SU}(3)$ pure gauge theories as well as the instanton liquid model (fitted to QCD). The results display remarkable similarity to the behavior of the $N=2$ theory, which indicates the potential significance of instanton effects.

\section{II. $N=1$ SUPERSYMMETRIC YANG-MILLS THEORY}

In this section, we will consider $N=1 \mathrm{SYM}$ theory for which the exact $\beta$ function is known. We will demonstrate that the exact coupling reproduces the assumptions underlying NDA. In particular, we will show that two relevant scales can be defined, the first at which the holomorphic coupling blows up and the second higher scale at which the true physical coupling becomes non-perturbative. The crucial distinction [4] is between the holomorphic (or "Wilsonian") coupling, which runs only at one-loop, and the "physical" one particle irreducible (1PI) coupling which receives corrections at all orders in perturbation theory. We will argue that the true scale of the strong interactions is the latter.

We first define the different scales. The quantity which is generally used to construct the holomorphic superpotential is defined by

$$
\Lambda_{D S}=\mu e^{-8 \pi^{2} / b_{0} g_{h}^{2}(\mu)}
$$

where $g_{h}^{2}$ is the Wilsonian coupling constant, defined as the coefficient of the gauge kinetic operator in the Lagrangian when written in a manifestly holomorphic form. Notice that although $g_{h}$ is scheme dependent, $\Lambda_{D S}$ is not. This is because the overall coefficient in Eq. (1), which we take to be 1 in dimensional reduction ( $\overline{\mathrm{DR}})$ [5], also changes accordingly.

Another useful scale to define is $\Lambda_{\infty}$, which we define as the scale at which the physical coupling becomes nonperturbative according to the exact Novikov-ShifmanVainshtein-Zakharov (NSVZ) $\beta$ function

$$
\Lambda_{\infty}=\mu\left(\frac{8 \pi^{2}}{g^{2}(\mu) N}\right)^{1 / 3} e^{-8 \pi^{2} / b_{0} g^{2}(\mu)}=\left(\frac{8 \pi^{2}}{N}\right)^{1 / 3} \Lambda_{D S}
$$

where we have used the relation between the holomorphic and physical 1PI coupling [6] in the last equality. Notice that according to NSVZ evolution the coupling constant never blows up. Instead it reaches a maximum at $\mu \simeq(1.39) \Lambda_{\infty}$ where $N g^{2} / 8 \pi^{2}=1$ and where perturbation theory breaks down. Notice also that the scale $\Lambda_{2-\text { loop }}$, defined by truncating the renormalization group (RG) beyond two loops (where it is anyway scheme dependent), differs from $\Lambda_{\infty}$ only by a factor $\left(1+g^{2} N / 8 \pi^{2}\right)^{1 / 3}$.

This relation between the Wilsonian and 1PI couplings was originally obtained by NSVZ [6] by considering the $S U(N)$ SYM instanton amplitude, which is proportional to

$$
A_{\text {inst }}=\mu^{3 N} \frac{e^{-8 \pi^{2} / g^{2}(\mu)}}{g^{2 N}(\mu)} \equiv \mu^{3 N} e^{-8 \pi^{2} / g_{h}^{2}(\mu)}=\Lambda_{D S}^{3 N} .
$$

The relation between $g_{h}^{2}$ and $g^{2}$ is also simply obtained by considering the nontrivial Jacobian which occurs when going from holomorphic to canonical gauge fields [7]. The Jacobian can be evaluated by using the Konishi anomaly together with the known fact that the beta function vanishes beyond 1-loop in $N=2$ SYM theory.

The important thing to notice in the two scales we have defined is that they differ by a reasonable factor, namely $\left(8 \pi^{2} / N\right)^{1 / 3}$, which is about 3 for low $N$.

Now we turn to naive dimensional analysis, which was applied to supersymmetric theories in Ref. [2] and applied in various model-building studies [8-13] to estimate nonperturbative contributions to the Kähler potential. According to NDA, operators composed of fields which are noncanonically normalized (with a coefficient of their kinetic term $\left.1 / g^{2}\right)$ are expected to have expectation values which scale according to the power of $\Lambda_{N D A}$ given by the dimension of the operator, with no additional factors of $4 \pi$. This can be derived by requiring that all orders in the loop expansion (with a cutoff) give comparable contributions to operators [1] or directly by rescaling fields in a suitably defined Wilson effective action [2]. Notice that in a supersymmetric theory the loop expansion is not a power series in the holomorphic coupling $g_{h}^{2}$ (there are $\ln g_{h}^{2}$ terms). It is the physical 1PI coupling which controls the loop expansion and which will turn out to be of order $4 \pi$ at the NDA scale. So, in order to use the picture of the second of Ref. [2], it is more appropriate to use a scheme for the Wilson effective action where the gauge coupling is not holomorphic (see for example Ref. [7]). The interesting thing in supersymmetric theories is that the scale $\Lambda$ which determines the overall coefficient of higher dimension operators (up to factors of order unity) can actually be determined. This is because there is an exact result to determine the scale, namely the gaugino condensate.

In Refs. [15,16,5], the gaugino condensate in SUSY $\mathrm{SU}(N)$ was obtained by considering first the theory with $N$ -1 flavors broken through the Higgs mechanism far out along a flat direction. The theory is then weakly interacting and a reliable calculation of the superpotential from instantons can be performed. Once this superpotential is obtained, the $N-1$ flavors are given a large mass, and the gluino condensate in the low-energy SYM theory is evaluated via the Konishi anomaly. The result is

$$
\left\langle\lambda_{a} \lambda_{a}\right\rangle=32 \pi^{2} \Lambda_{D S}^{3}
$$


where $\lambda$ is noncanonically normalized, with coefficient of its kinetic term $1 / g^{2}(\mu)$, and $\Lambda_{D S}$ agrees with our definition above if $g(\mu)$ is the $(\overline{\mathrm{DR}})$ coupling. ${ }^{1}$

On the other hand, according to NDA, the gaugino condensate should be

$$
\left\langle\lambda_{a} \lambda_{a}\right\rangle \sim \Lambda_{N D A}^{3} .
$$

It should be noted that NDA does not usually incorporate large $N$ factors, but it can be easily done. In the noncanonical normalization, the gauge propagator is $\sim g^{2}(p) / p^{2}$. By NDA, the condensate is going to be saturated by loop momenta of order $\Lambda_{N D A}$, so that

$$
\begin{aligned}
\left\langle\lambda_{a} \lambda_{a}\right\rangle & \sim N^{2} \int \frac{p^{2} d p^{2}}{16 \pi^{2}} \frac{g^{2}(p)}{p} \sim \frac{N^{2} g^{2}\left(\Lambda_{N D A}\right) \Lambda_{N D A}^{3}}{16 \pi^{2}} \\
& =N \Lambda_{N D A}^{3} .
\end{aligned}
$$

The qualitative scaling of Eq. (7) is also reproduced with the use of gap equations [14].

By comparing the above to the exact result in Eq. (4) one gets

$$
\Lambda_{N D A}=\left(\frac{32 \pi^{2}}{N}\right)^{1 / 3} \Lambda_{D S}
$$

which, within an $\mathcal{O}(1)$ factor, coincides with $\Lambda_{\infty}$. This is precisely what one would want to find; $\Lambda_{N D A}$ corresponds to the scale at which the physical coupling becomes nonperturbative and the description of the theory must change. Indeed, accounting also for large $N$ factors, the loop expansion parameter of SYM theory should be $x \sim N g^{2} /(4 \pi)^{2}$. Thus, by Eq. (2) we have that $\Lambda_{N D A}$, at which $x \sim 1$, essentially coincides with $\Lambda_{\infty}$. In other words, we find out that NDA reproduces both the $4 \pi$ 's and large $N$ behavior of the instanton calculation at weak coupling. Given that it was the physical $g(\mu)$ which appeared in the propagator, it was natural to expect the loop counting parameter to be the 1PI rather than Wilsonian coupling. Notice indeed that the Wilsonian expansion parameter at the NDA scale is roughly

$$
\frac{N g_{W}^{2}}{8 \pi^{2}} \sim \frac{1}{1+\ln \left(8 \pi^{2} / N\right)}
$$

which for small $N$ may look perturbative.

As an aside, we comment on the well-known fact $[6,17,5]$ that the direct calculation of $\langle\lambda \lambda\rangle$ in pure, strongly coupled, SYM theory disagrees with the weak coupling result calculated in the Higgs phase. In particular even the large $N$ behavior is different,

${ }^{1}$ In Ref. [5], the scheme independent factor $1 / g^{2 N}$ was never explicitly displayed; if included, it would combine together with the $g(\mu)$ in the exponent to give the instanton amplitudes in terms of $g_{h}^{2}$.

$$
\langle\lambda \lambda\rangle_{\text {strong }}=\frac{1}{N}\langle\lambda \lambda\rangle_{\text {weak }},
$$

NDA gives a result in agreement with weak coupling methods. The origin of the discrepancy is not yet established. One possibility is that while at weak coupling instantons presumably saturate the condensate, there are new effects, other than instantons, in the strong coupling regime. Quite interestingly, as a result of the space-time independence of the $N$-point $\lambda \lambda$ correlator, these effects should be important even at short distance in the strong coupling regime. However, since the scale at which the theory became nonperturbative was adequately accounted for in perturbation theory, both instantons and these additional nonperturbative effects seem irrelevant to establishing the NDA scale. Finally, another possibility, recently proposed by Kovner and Shifman, is that there exists a chirally symmetric phase of SYM theory [18]. In this way the strong coupling result, interpreted as a weighted average over chirally symmetric and asymmetric vacua, is bound to be the lower.

The obvious question now is how to extrapolate these lessons to real QCD. In QCD, we know that a 2 loop calculation, although it goes in the right direction, does not change the QCD scale sufficiently to account for NDA. In fact, the relation between scales in this case is given as

$$
\Lambda_{2 \text {-loop }} \simeq\left(\frac{3.2 \pi^{2}}{g^{2}}\right)^{0.33} \Lambda_{1-\text { loop }}
$$

where 5 flavors have been assumed (but the result is rather independent of that). By running from $\mu=M_{Z}$, the above equation changes the one-loop QCD scale of about $100 \mathrm{MeV}$ to its two-loop value of about $250 \mathrm{MeV}$. This is still about 4 times smaller than the "observed" NDA scale of QCD. As we said before, higher loop effects, or scheme dependence, in the perturbative definition of $\Lambda_{N D A}$ could amount to an $\mathcal{O}(1)$ factor. So the "big" factor in QCD is puzzling. In the next section we will argue that additional corrections could come from instantons. This gives rise to the obvious question of why the instanton effects do not affect the "exact" coupling of $N=1$ SYM theory. We will address this issue in the concluding section. The discussion of this section seems to suggest that additional effects, if present, are not very important in determining the strong scale, as the condensate is well estimated perturbatively.

Finally we briefly comment on the relation, suggested by NDA, between the hadron masses and their sizes, as determined by the confinement scale. Consider for instance the elastic scattering of a spin zero glueball $S$ of mass $\sim \Lambda_{N D A}$. By NDA we expect the quartic coupling to be $\mathcal{O}\left(16 \pi^{2}\right)$, so that the elastic cross section is $\sim 4 \pi\left(4 \pi / \Lambda_{N D A}\right)^{2}$. This result can be interpreted as due to the collision of two hard objects of radius $4 \pi / \Lambda_{N D A}$. This remains true also at large $N$ though there the mesons are weakly interacting, and the suitable factor of $N$ must be factored out in the cross section. It should be noted that from this point of view, the coincidence of $\Lambda_{Q C D}$, the scale at which the perturbative gauge coupling blows up, and the confinement scale, $250 \mathrm{MeV}$ $\approx 2 \Lambda_{N D A} / 4 \pi$, is merely coincidental. The first scale seems 
to have no physical meaning, though it can of course be defined from the two-loop $\beta$-function.

What we have learned about NDA in SYM theory can be useful in particle physics models where the strong interaction scale $\Lambda$ itself depends on some modulus $X$. In the models of Refs. [11-13] $X$ gives mass to all $S U(N)$ flavors, so that below the scale $X$ the effective gauge theory is just SYM theory. The strong dynamics then generates a superpotential for $X$ via gaugino condensation. In Refs. $[11,12]$ the effective superpotential is $W_{e f f}=\Lambda_{D S}^{3}(X)=\Lambda^{2} X$, where $\Lambda_{D S}(X)$ and $\Lambda$ are respectively the holomorphic scales in the lowand high-energy theories. The resulting potential is very flat, and an estimate of the non-perturbative Kähler potential can be crucial. The only way we can do that at the moment is by NDA. In Ref. [12] $X$ is the inflaton so that the size and sign of these corrections can have a crucial impact on the slow roll. We have argued that NDA should apply for SYM. Denoting by $\Lambda_{N D A}(X)$ and $\Lambda_{D S}(X)$ the scales of the low energy theory the effective Lagrangian is [13]

$$
\begin{gathered}
\mathcal{L}_{e f f}=\frac{1}{16 \pi^{2}}\left(\int d^{4} \theta c_{1} \Lambda_{N D A}(X)^{\dagger} \Lambda_{N D A}(X)\right. \\
\left.+\int d^{2} \theta \Lambda_{N D A}(X)^{3}+\text { H.c. }\right)
\end{gathered}
$$

where $c_{1}$ is expected to be $O(1)$. By writing the above in terms of the original holomorphic $\Lambda$ we have that the correction to the Kähler metric is

$$
\delta K_{X X^{\dagger}}=\frac{c_{1}}{9}\left(\frac{1}{4 \pi}\right)^{2 / 3}\left(\frac{\Lambda}{X}\right)^{4 / 3} .
$$

This scaling with $4 \pi$ can also be established by a direct diagrammatic analysis. For this purpose it is useful to parametrize $X=\langle X\rangle+\delta X$, where $\langle X\rangle$ is the $c$-number vacuum expectation value (VEV). Below the scale $\langle X\rangle$, where the messengers are integrated out, $\delta X$ couples effectively to the SYM theory via a one-loop effect

$$
\frac{1}{8 \pi^{2}} \int d^{2} \theta \frac{\delta X}{\langle X\rangle} W_{\alpha} W^{\alpha}+\cdots
$$

At second order in this interaction, we get a quantum correction to the $\delta X$ wave function

$$
\delta K_{X X^{\dagger}}=\left(\frac{1}{8 \pi^{2}}\right)^{3} \int_{0}^{\langle X\rangle^{2}} d p^{2} \frac{g^{4}\left(p^{2}\right)}{\langle X\rangle^{2}}
$$

where two powers of $1 / 8 \pi^{2}$ come from insertions of Eq. (15). For large $\langle X\rangle$ the leading contribution to Eq. (16) is perturbative, $\sim g^{4}(\langle X\rangle) /(4 \pi)^{6}$, and comes from integration at $p \sim\langle X\rangle$. Non-perturbative effects are estimated via NDA by the contribution at $p \sim \Lambda_{N D A}$ to Eq. (16). The result agrees with the estimate in Eq. (14). This effect becomes rapidly important when $\langle X\rangle$ is decreased.

It would be interesting to have information on the sign of this correction. Notice that $\delta K_{X X^{\dagger}}$ is proportional to the cor- relator $G_{\lambda}=\langle(\lambda \lambda)(\bar{\lambda} \bar{\lambda})\rangle$ at zero momentum. ${ }^{2}$ It is useful to consider the dispersive Källen-Lehman representation for $G_{\lambda}$ :

$$
G_{\lambda}\left(p^{2}\right)=\int_{0}^{\infty} d m^{2} \frac{m^{2} \sigma\left(m^{2}\right)}{p^{2}-m^{2}}
$$

where $\sigma\left(\mathrm{m}^{2}\right)$ is a positive definite function. ${ }^{3}$ Our basic point is that we can perform an operator product expansion (OPE) for $G_{\lambda}$ in the Euclidean $-p^{2} \gg \Lambda_{N D A}^{2}$ region. The lowest power correction corresponds to $\langle G G\rangle / p^{4} \sim \Lambda_{N D A}^{4} / p^{4}$. It is reasonable to expect that the corrections to $\sigma\left(m^{2} \gg \Lambda_{N D A}^{2}\right)$ scale in the same way. If that is the case, the leading nonperturbative contribution to $\delta K_{X X^{\dagger}} \propto G_{\lambda}\left(p^{2}=0\right)$ comes only from the region of integration at $m^{2} \sim \Lambda_{N D A}^{2}$. This gives more justification to the NDA estimate we did above. This also suggests that it is probably not unreasonable to assume that the effect is well described by summing just over the lowest resonances in $\sigma$. Under that assumption we conclude that $c_{1}>0$ in Eq. (14). This result has important implications for the models of Refs. $[11,12]$. There the correction with $c_{1}>0$ creates a potential that "pushes" the $X$ field towards the origin. This is not problematic for the inflationary models of Ref. [12], but it can destabilize the local vacuum of the gauge mediated scenarios of Ref. [11]. The condition to avoid the latter problem has already been discussed in Ref. [11]: it requires the scale $X$ to be above $10^{9}-10^{10} \mathrm{GeV}$.

\section{III. $N=2$ SUSY SU(2)}

For $N=2$ SUSY QCD the effective Lagrangian to leading order in a momentum expansion was derived in an exact solution by Seiberg and Witten [20]. Although the dynamics and physical fields are very different from QCD, one can nonetheless observe a similar puzzle; the effective coupling blows up at the point where according to the one-loop beta function one would get $g^{2} /(4 \pi)=\alpha=0.76$. This might have been thought to be a safely perturbative region, but in fact the instanton effects become large at this point; they induce a very strong interaction and make the use of the original formulation of the theory impossible.

This is seen from the exact result for the effective coupling

$$
\frac{8 \pi}{g^{2}(u)}=\frac{K\left(\sqrt{1-k^{2}}\right)}{K(k)}
$$

where $K$ is elliptic integral and the argument

\footnotetext{
${ }^{2} \delta K_{X X^{\dagger}}$ can be obtained by focusing on the correction to $F_{X} F_{X}^{\dagger}$ and by noticing that $F_{X}$ couples just to $\lambda \lambda$ in Eq. (15).

${ }^{3}$ Notice that $\sigma \sim g^{4}\left(m^{2}\right)$, at $m \gg \Lambda_{N D A}$, giving an apparent quadratic divergence. This is regulated by performing suitable matching to the above theory at the scale $\langle X\rangle$. Above this scale there is just the logarithmic divergence of the $X$ wave function.
} 


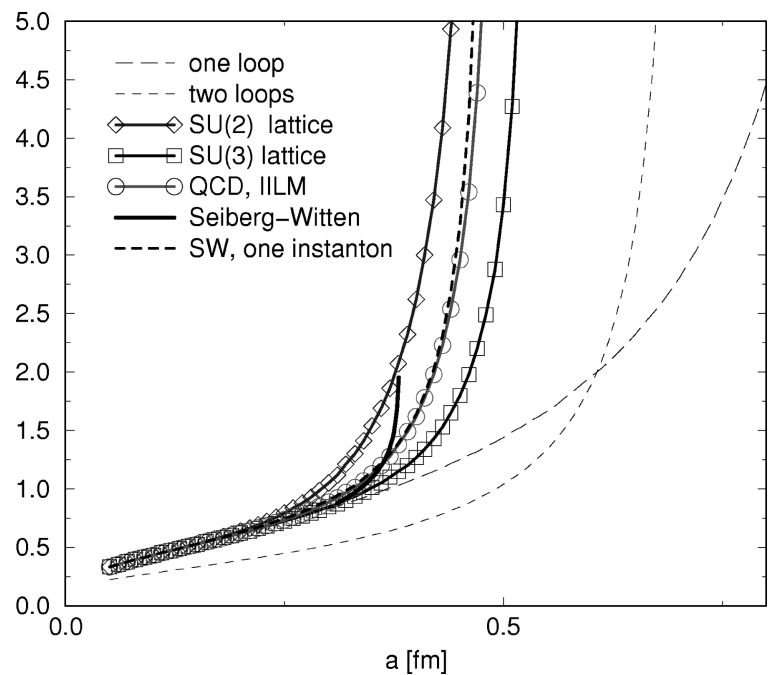

FIG. 1. The effective charge $b g_{\text {eff }}^{2}(\mu) / 8 \pi^{2}$ ( $b$ is the coefficient of the one-loop beta function) versus normalization scale $\mu$ (in units of its value at which the one-loop charge blows up). The thick solid line corresponds to exact solution [20] for the $N=2$ SYM theory; the thick dashed line shows the one-instanton correction. Lines with symbols (as indicated on figure) stand for $N=0$ QCD-like theories, $\mathrm{SU}(2)$ and SU(3) pure gauge ones and QCD itself. Thin longdashed and short-dashed lines are one- and two-loop results.

$$
k^{2}=\frac{u-\sqrt{u^{2}-4 \Lambda^{4}}}{u+\sqrt{u^{2}-4 \Lambda^{4}}}
$$

is a function of the gauge invariant vacuum expectation of the squared scalar field,

$$
u=\frac{1}{2}\left\langle\phi^{2}\right\rangle=\frac{a^{2}}{2}+\frac{\Lambda^{4}}{a^{2}}+\cdots,
$$

and $a$ is just its VEV. For large $a$ there is a weak coupling expansion which includes instanton effects ${ }^{4}$

$$
\frac{8 \pi}{g^{2}(u)}=\frac{2}{\pi}\left[\log \left(\frac{2 a^{2}}{\Lambda^{2}}\right)-\frac{3 \Lambda^{4}}{a^{4}}+\cdots\right] .
$$

The exact coupling blows up at $u=2 \Lambda^{2}$, which means that the factor between the exact strong interaction scale and the perturbative one is in this theory $\Lambda_{\infty}=2^{3 / 2} \Lambda_{D S}$. Actually this is the ratio of the scale $\sqrt{u}$ to the scale $a$ at which the perturbatively evolved coupling (one-loop) blows up. If one were to account for the next term in the expansion of $u$, the ratio of scales is reduced to $\sqrt{2} \sqrt{2+\sqrt{2}}$. The fact that instanton effects can be important at such a high scale was anticipated in Ref. [3] and is presumably due to the significance of the prefactor in instanton calculations.

The behavior is shown in Fig. 1, where we have included both a curve which shows the full coupling (thick solid line), as well as a curve which illustrates only the one-instanton

\footnotetext{
${ }^{4}$ It should be noted that the first terms in this expansion have been explicitly verified in instanton calculations [21].
}

correction (thick dashed one). Because we will want to compare the running of the coupling in different theories, we have plotted $b g^{2} / 8 \pi^{2}$ ( $b=4$ in this case is the one-loop coefficient of the beta function) and measure all quantities in units of $\Lambda_{D S}$, so that the one-loop charge blows out at 1 . The meaning of the scale can therefore be determined by what enters in the logarithm. We have plotted the exact result against $\phi$ rather than $a$.

Note the very rapid change of the coupling induced by instantons. It is also of interest that the full multi-instanton sum makes the rise in the coupling even more radical than with only the one-instanton correction incorporated. It is also interesting to observe that at the scale where the true coupling blows up, the perturbatively evolved coupling is still not very large. Individually, the perturbative logarithmic and instanton corrections are well defined at this region: however, they cancel each other in the inverse charge. This is encouraging from the point of view of developing a consistent expansion for the instanton corrections. The rapid rise in the coupling is also encouraging in that it ensures that perturbation theory is valid almost to the point where it blows up. For a consistent picture of QCD, in which perturbation theory still appears to be applicable at the $c$-quark scale, while the theory is nonperturbative at $1 \mathrm{GeV}$, such a dramatic effect is essential.

So the basic lessons seems to be, first, that instantons can lead to a discrepancy between the scales at which the perturbative evolution of the coupling blows up and the nonperturbative coupling blows up. Second, the rise in the coupling is very dramatic. Third, the coincidence between $f_{\pi}$ and $\Lambda_{Q C D}$ seems to be just that. Fourth, even though we are in a weak coupling regime where the instantons should be dilute, there is a notable difference between the rise in the coupling due to one instanton and due to the full instanton sum; multiinstantons are also important.

\section{QCD}

Having learned what we can from exact SUSY results, we now turn to ordinary QCD. We know that naive dimensional analysis appears to work, but with the dimensional scale which sets the cutoff and which suppresses higher dimensional operators set by $1 \mathrm{GeV}$. As the theory is not supersymmetric, we cannot derive this dimensional scale as we did with the gluino condensate. Nonetheless, sum rules [22] and lattice simulations [23] yield, consistently, a quark condensate $\langle\bar{q} q\rangle \sim(250 \mathrm{MeV})^{3}$. On the other hand, NDA suggests

$$
\langle\bar{q} q\rangle=\frac{\Lambda_{N D A}^{3}}{16 \pi^{2} Z\left(\Lambda_{N D A}\right)} \sim \frac{(1 \mathrm{GeV})^{3}}{16 \pi^{2} Z\left(\Lambda_{N D A}\right)}
$$

where $Z$ is the multiplicative renormalization of the composite operator $\bar{q} q$. Equation (22) agrees with the phenomenological value of the condensate if $Z\left(\Lambda_{N D A}\right) \sim 1$. This requirement is not inconsistent as $Z$ does not run very fast.

The question then is where this scale arises in terms of the theory of the fundamental fields, the quarks and gluons. We know that the parton model is limited from below by the 1 
$\mathrm{GeV}$ scale, but perturbative QCD seems well-behaved at this scale. For the $N=1$ coupling, we know we can estimate the dimensional analysis scale using only the leading two terms in perturbation theory. We also know this is inadequate in QCD, as the subleading term changes the QCD scale from about $100 \mathrm{MeV}$ to $250 \mathrm{MeV}$; higher order terms should not change this substantially.

So it seems that if the NDA scale of $1 \mathrm{GeV}$ is to have a physical meaning, it is probably due to nonperturbative effects in the evolution of the coupling. It is possible that instantons are the only nonperturbative effect which is substantial above the confinement scale. This was the original CDG suggestion [3]. For a review of subsequent developments and phenomenological fits in support of this hypothesis with allowance for inter-instanton interactions (the "instanton liquid model") one may consult the review [24].

For the purpose of this discussion we restrict ourselves to the one instanton effect (though for QCD the distribution allows for instanton interactions), a single-instanton correction to the effective action of some smooth background gauge field $G_{\mu \nu}$. The external field is supposed to be normalized at some normalization scale $\mu$, and CDG have proposed to include all instantons with size $\rho<\rho_{\max }=1 / \mu$. The effective charge is then defined as

$$
\begin{aligned}
\frac{8 \pi^{2}}{g_{\text {eff }}^{2}(\mu)}= & b \ln \left(\frac{\mu}{\Lambda_{\text {pert }}}\right)-\frac{4 \pi^{2}}{\left(N_{c}^{2}-1\right)} \\
& \times \int_{0}^{\rho_{\max }} d n(\rho) \rho^{4}\left(\frac{8 \pi^{2}}{g_{\text {eff }}^{2}(\rho)}\right)^{2}
\end{aligned}
$$

where $b=11 N_{c} / 3-2 N_{f} / 3$ is the usual one-loop coefficient of the beta function, and $d n(\rho)$ is the distribution of instantons (and anti-instantons) over size.

The instanton density is semiclassically calculable only at small $\rho$ where it is very small, and therefore for high scales the instanton correction is tiny, $\sim(\Lambda / \mu)^{b}$. At larger $\rho$ the instanton size distribution (known from lattice studies and model-dependent calculations) has a strongly peaked shape, with the peak at $\rho \sim .2-.3 \mathrm{fm}[19]$. As soon as $\rho_{\max }=1 / \mu$ becomes close to the position of the peak, the CDG effective charge (23) blows up.

Of course in order to establish the magnitude of the effect of instantons one needs to know the instanton distribution. In Fig. 1 we compare three QCD-like theories. The first two are pure gauge $\mathrm{SU}(2)$ and $\mathrm{SU}(3)$ where the distributions are obtained by cooling of lattice configurations from [25] and [26] respectively. There are other recent lattice works for $\mathrm{SU}(2)$ and SU(3) which we have not included, e.g. [27] and [28]. For QCD with three light flavors, we use the interacting instanton liquid model (IILM) [29] to provide a model distribution.

The two-loop result (shown by the thin short-dashed line) is the same for any pure gauge theory (with minor modifications for QCD which are not shown). As we have mentioned, the two-loop running raises the scale at which the coupling gets big, but does not generate a sufficiently large scale. With instantons present, the coupling blows up at a somewhat higher scale, where perturbation theory looks naively still valid. It is perhaps a little surprising that all the theories including instantons look so similar, and that furthermore the instanton effects are so similar to that for $N=2$ SUSY QCD. Although this is probably accidental, it does establish that instantons can generate a somewhat higher scale, and that the theory looks perturbative until almost reaching this scale (that is the coupling rises very rapidly). One may further speculate by analogy to the $N=2$ theory that multi-instanton effects yield an even more substantial rise in the coupling.

\section{DISCUSSION AND CONCLUSIONS}

In conclusion, we have argued that in $N=1$ pure gauge theory the analogue of the "chiral Lagrangian" scale corresponds to the scale where the physical (1PI) coupling becomes of order $4 \pi$. We had two benefits from considering the supersymmetric theory; the NDA scale is determined by the gluino condensate and furthermore the exact beta function is known. Our argument is made by comparing the known exact value of the gluino condensate to a "naive dimensional analysis estimate" and by evaluating the exact coupling at this scale. This is a useful conclusion for applications to supersymmetric model building $[2,8,10-13]$. The lessons for QCD are less obvious. In QCD the chiral Lagrangian scale $\Lambda_{N D A}$ is in fact somewhat larger $(\sim 1 \mathrm{GeV})$ than the scale $\Lambda_{Q C D} \sim 250 \mathrm{MeV}$ which is associated with the perturbative coupling. It was pointed out, almost two decades ago [3], that small instantons can lead to a precocious breakdown of perturbation theory, i.e. at scale where $\alpha / 4 \pi$ is somewhat less than unity. It is an important question how instanton effects can be relevant for QCD but not relevent for $N=1$ supersymmetric theories.

Consider, for example, $N=0 \mathrm{SU}(3)$ with three flavors and $N=1 \mathrm{SU}(3)$ with no flavors. These theories have identical $b_{0}$ and numerically very similar $b_{1}$. Since the perturbative scaling is therefore practically the same, we need to understand whether it is possible that instanton effects can be important for the first theory at a scale in which they can be neglected in the second.

To address this question, we first need to remind ourselves how instantons can affect the coupling. The naive answer is that they do not since the exact beta function is given without including instanton corrections to the running. In essence supersymmetry protects against contributions from small instantons. However, this answer is inadequate since we already know that the dominant instanton contribution will only affect the coefficient of a multifermion operator. This only contributes to the renormalization of the gauge coupling in the presence of a nonzero gluino condensate, which is only relevant in the infrared and would not be included in the definition of the beta function.

Now let us compare our two theories in more detail. We need to determine the normalization by which we will compare the theories. This is straightforward as we can take identical gauge coupling values in the ultraviolet for the two theories in a safely perturbative regime. Subsequently, running down in energy, both couplings will run according to perturbation theory essentially identically. Actually there is a caveat that the coupling we generally use in QCD is the 
modified minimal subtraction $(\overline{\mathrm{MS}})$ coupling whereas the ( $\overline{\mathrm{DR}}$ ) scheme is used in the $N=1$ theory. However, one can explicitly check that the difference in couplings is sufficiently small that it does not affect the argument below.

Now for the $N=1$ theory we know the NDA scale by the arguments given previously. In fact we know it is the scale at which the coupling blows up, which is about $250 \mathrm{MeV}$.

Let us consider the fermion condensates and their evolution in the two theories (we mean here the condensates of the fields that are canonically normalized at each scale). At the scale where $g^{2} \sim 1$ the two condensates are roughly the same since $\langle\lambda \lambda\rangle=\left(\Lambda_{N D A}^{N=1}\right)^{3}=(250 \mathrm{MeV})^{3}$ and $\langle\bar{q} q\rangle$ $=(1 \mathrm{GeV})^{3} / 16 \pi^{2} \simeq(250 \mathrm{MeV})^{3}$. Now let us compare their values at $1 \mathrm{GeV}$. The gluino condensate scales inversely to the gauge coupling squared and is therefore $(250 \mathrm{MeV})^{3} / g^{2}(1 \mathrm{GeV}) \simeq(250 \mathrm{MeV})^{3} / 4 \pi$. This is smaller than the quark condensate which is scaling with the mass anomalous dimension, that is, much more slowly, and is therefore still about $(250 \mathrm{MeV})^{3}$. That is, the QCD condensate is significantly larger. It is therefore not inconsistent for instantons to be significant at $1 \mathrm{GeV}$ in QCD, but not in the comparable supersymmetric theory. Although this does not establish the importance of instantons for QCD, it is reassuring.

In order to probe the possible relevance of instanton effects, we studied the effective gauge coupling in the $N=2$ $S U$ (2) SYM theory. There also we found that instanton effects lead to a precocious explosion of the coupling. We compared the effective charge as a function of the adjoint VEV in $N=2$ with the effective charge of $N=0$ gauge theories obtained by smearing over small instanton effects. We used the instanton liquid model and lattice data to estimate the instanton density. We found a suggestive similarity (see
Fig. 1) between these phenomenological models and the exact $N=2$ case. Furthermore, the generated scales are very close, and for QCD it is not so far from $1 \mathrm{GeV}$, the right phenomenological value. All this seems to support the instanton scenario of the generation of this scale for QCD, although it does not exclude other nonperturbative effects.

In summary, we have looked at potential implications of SUSY theories for our understanding of low energy QCD. Because the phase structure of supersymmetric theories is so different, we restricted our attention to the question of obtaining a consistent picture of the boundary of the perturbative domain. We have not addressed the issue of chiral symmetry breaking and confinement (aside from mention of the geometric relation between the confinement scale and the chiral Lagrangian scale). We have also not addressed the possible discrepancies in the effective coupling which can appear depending on the correlation function at low energy. We have argued that in fact the fundamental QCD scale of the theory is more readily associated with the blowup of the physical coupling, and that this is more likely to be the higher chiral Lagrangian scale. We also note that the separation of scales due to instantons argues against a standard large- $N$ interpretation of QCD, for which instantons would not be important.

\section{ACKNOWLEDGMENTS}

We acknowledge useful conversations with N. ArkaniHamed, M. Beneke, C. Callan, A. Cohen, D. Finnell, H. Georgi, R. Jaffe, K. Johnson, D. Kaplan, M. Luty, P. Nason, J. Negele, A. Nelson, M. Porrati, M. Strassler and F. Wilczek. L. R. thanks Princeton University and the Institute for Advanced Study for their hospitality during the course of this work.
[1] H. Georgi and A. Manohar, Nucl. Phys. B234, 189 (1984).

[2] M. A. Luty, Phys. Rev. D 57, 1531 (1998); A. G. Cohen, D. B. Kaplan, and A. E. Nelson, Phys. Lett. B 412, 301 (1997).

[3] C. G. Callan, R. Dashen, and D. J. Gross, Phys. Rev. D 17, 2717 (1978).

[4] M. Shifman and A. Vainshtein, Nucl. Phys. B277, 456 (1986).

[5] D. Finnell and P. Pouliot, Nucl. Phys. B435, 225 (1995).

[6] V. A. Novikov, M. A. Shifman, A. I. Vainshtein and V. I. Zakharov, Nucl. Phys. B229, 381 (1983); B260, 157 (1985); Phys. Lett. 166B, 334 (1986).

[7] N. Arkani-Hamed and H. Murayama, Report No. LBNL40460, hep-th/9707133.

[8] D. Kaplan, F. Lepeintre, and M. Schmaltz, Phys. Rev. D 56, 7193 (1997).

[9] A. Cohen, D. Kaplan, and A. Nelson, Phys. Lett. B 388, 588 (1996).

[10] A. E. Nelson and M. J. Strassler, Phys. Rev. D 56, 4226 (1997)

[11] H. Murayama, Phys. Rev. Lett. 79, 18 (1997); S. Dimopoulos, G. Dvali, R. Rattazzi, and G. Giudice, Nucl. Phys. B510, 12 (1998)
[12] S. Dimopoulos, G. Dvali, and R. Rattazzi, Phys. Lett. B 410, 119 (1997).

[13] M. Luty, Phys. Lett. B 414, 71 (1997); M. Luty and J. Terning, Phys. Rev. D 57, 6799 (1998).

[14] T. Appelquist, A. Nyffeler, and S. B. Selipsky, Phys. Lett. B 425, 300 (1998).

[15] M. Shifman and A. Vainshtein, Nucl. Phys. B496, 445 (1988).

[16] A. Yu. Morozov, M. A. Olshanetskii, and M. Shifman, Nucl. Phys. B304, 291 (1988).

[17] D. Amati, K. Konishi, Y. Meurice, G. C. Rossi, and G. Veneziano, Phys. Rep. 162, 169 (1988).

[18] A. Kovner and M. Shifman, Phys. Rev. D 56, 2396 (1997).

[19] E. V. Shuryak, Phys. Lett. 107B, 103 (1981); Nucl. Phys. B203, 93 (1982).

[20] N. Seiberg and E. Witten, Nucl. Phys. B426, 19 (1994).

[21] N. Dorey, V. Khoze, and M. P. Mattis, Phys. Rev. D 54, 2921 (1996); F. Fucito and G. Travaglini, ibid. 55, 1099 (1997); N. Dorey, V. Khoze, and M. P. Mattis, Phys. Lett. B 390, 205 (1997).

[22] J. Bijnens, J. Prades, and E. de Rafael, Phys. Lett. B 348, 226 (1995); M. Jamin and M. Münz, Z. Phys. C 66, 633 (1995). 
[23] R. Gupta and T. Bhattacharya, Phys. Rev. D 55, 7203 (1997).

[24] T. Schäfer and E. V. Shuryak, Rev. Mod. Phys. 70, 323 (1998); Annu. Rev. Nucl. Part. Sci. 47, 359 (1997).

[25] C. Michael and P. S. Spencer, Nucl. Phys. B (Proc. Suppl.) 42, 261 (1995).
[26] P. de Forcrand et al., Nucl. Phys. B (Proc. Suppl.) 63, 549 (1998).

[27] T. DeGrand, A. Hasenfratz, and D. Zhu, Nucl. Phys. B (Proc. Suppl.) B478, 349 (1996).

[28] D. Smith et al., Nucl. Phys. B (Proc. Suppl.) 63, 588 (1998).

[29] E. V. Shuryak, Phys. Rev. D 52, 5370 (1995). 